apuntesuniversitarios.upeu.edu.pe

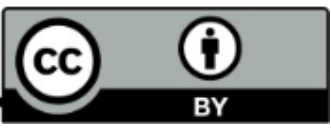

Apuntes Universitarios, 2020: 10(4), octubre-diciembre

ISSN: 2304-0335 DOI: https://doi.org/10.17162/au.v10i4.487

\title{
Validez de constructo y confiabilidad de un instrumento para evaluar la atención a la diversidad estudiantil en danza
}

\author{
Construct validity and reliability of an instrument to evaluate attention to \\ student diversity in dance \\ Yeny Avila-García ${ }^{1 \mathrm{a}}$ y Sergio Raúl Herrera-Meza² \\ Universidad Autónoma de Chihuahua, México ${ }^{1}$ \\ Centro Universitario CIFE, México ${ }^{2}$ \\ ORCID ID: https://orcid.org/0000-0002-4279-3411 ${ }^{1}$ \\ iD ORCID ID: https://orcid.org/0000-0003-2605-4878²
}

Recibido: 19 de diciembre de 2019

Aceptado: 11 de junio de 2020

\begin{abstract}
Resumen
El objetivo del presente trabajo fue realizar el estudio de validez de constructo y confiabilidad del instrumento CEADED que evalúa la atención a la diversidad entre estudiantes de danza a nivel universitario. Se realizó un estudio instrumental con base en los siguientes pasos: 1) analizar la validez de constructo mediante el empleo del análisis factorial; y 2) analizar la confiabilidad del instrumento. Se aplicó el instrumento a una muestra de 453 estudiantes de carreras profesionales en danza procedentes de diversas universidades de México, y se realizó el análisis factorial mediante el método de extracción de factorización de ejes principales iterado con rotación Oblimin Directo. El análisis factorial mostró ocho factores que explicaron el $65.260 \%$ de la varianza y la confiabilidad fue de un Alfa de Cronbach de 0.957.
\end{abstract}

Palabras clave: diversidad, confiabilidad, desarrollo sostenible, inclusión educativa, sociedad del conocimiento, validez de constructo.

\begin{abstract}
The objective of this work was to perform the construct validity and reliability study of the CEADED instrument that evaluates attention to diversity among dance students at the university level. An instrumental study was carried out based on the following steps: 1) determine the valid ity of the construct through the use of factor analysis; and 2) analyze the reliability of the instrument.
\end{abstract}

${ }^{\mathrm{a} C}$ Correspondencia al autor

E-Mail: yavila@uach.mx 
The instrument was applied to a sample of 453 dance professional students from various universities in Mexico, and the factorial analysis was carried out using the method of extraction of factorization of principal axes iterated with Oblimin Direct rotation. The factorial analysis showed eight factors that explained $65.260 \%$ of the variance and the reliability was of a Cronbach's alpha of 0.957 .

Keywords: attention to diversity, reliability, sustainable development, educational inclusion, knowledge society, construct validity.

\section{Introducción}

En las últimas décadas, el tema de la inclusión y atención a la diversidad ha dado lugar a cambios importantes en el ámbito educativo universitario en cuanto al modo de abordar los procesos de enseñanza y aprendizaje. La forma de planificar y concebir este proceso ha dado un vuelco de 360 grados al poner en tela de juicio la enseñanza tradicional, preponderantemente selectiva y, en algunos casos discriminatoria (Agreda, Hinojos y Sola, 2016).

De ahí, la necesidad de establecer procesos formativos más inclusivos y equitativos que permitan hacer frente a cualquier conducta desigual (Ramos-Carranza et al., 2015). Desde el enfoque socioformativo es posible brindar atención a la educación para el desarrollo sostenible, refiriendo al concepto, como aquel desarrollo capaz de satisfacer las necesidades actuales de manera sostenida en el tiempo, que pueda mantenerse sin comprometer los recursos y respetando la diversidad en todas sus expresiones (Severiche-Sierra, Gómez-Bustamante, y Jaimes-Morales, 2016) y promover el talento humano, lo que consiste en lograr que todas las personas alcancen sus metas independientemente de su condición física, sociocultural, cognitiva o económica (Tobón, 2017).

Lo anterior, se relaciona con el abordaje de la diversidad en el campo del arte danzario, lo cual ha resultado algo complicado. No ha sido fácil romper con un proceso formativo que, por tradición, ha ambicionado formas perfectas y virtuosas en los cuerpos de los bailarines (Montes, 2017). La búsqueda de una danza transformada y propositiva implica aceptar alternativas más comprometidas (Brozas-Polo y Vicente-Pedraz, 2017) con el desarrollo del talento de las personas (Tobón, 2017) y no solamente con el logro de atractivas formas corporales. Para ello, es importante evaluar los componentes que forman parte de los procesos formativos de los profesionales de la danza para verificar el grado en que promueven, favorecen o dificultan la inclusión a la diversidad. 
Existen diversos instrumentos para evaluar la atención a la diversidad. Uno de ellos, es el cuestionario AVACO-EVADIE (Análisis de Variables de Contexto para Evaluación Sistemas Educativos-Evaluación de la Atención a la Diversidad como Dimensión Educativa en las Instituciones Escolares), mismo que fue elaborado en el contexto de España y está compuesto por una serie de indicadores que evalúan el comportamiento de los profesores, directores, inspectores y orientadores ante la diversidad: recursos, centro, concepto de normalidad, concepto de diversidad, intervención del centro, diagnóstico de los alumnos, currículo y nivel de rendimiento (BiencintoLópez et al., 2009).

Otro instrumento también elaborado en España, es el cuestionario ACADI (Autoevaluación de Centros para la Atención a la Diversidad desde la Inclusión) que está compuesto por cuatro dimensiones: contexto escolar, recursos, proceso educativo y resultados (Arnaiz y Guirao, 2015). Por su parte el CEEIU (Cuestionario para Evaluar la Educación Inclusiva en la Universidad), es un instrumento que evalúa el constructo "educación inclusiva universitaria" y fue diseñado en el contexto de Ecuador. Está formado por las dimensiones: conocimiento, cultura y política institucional, alumnos, formación-desarrollo profesional y enseñanza (De la Herrán, Paredes, y Monsalve, 2017).

Estos instrumentos tienen en común que: a) están enfocados a la educación básica y media; b) coinciden en cuanto a los propósitos y las dimensiones para medir la atención a la diversidad o inclusión educativa; y c) cuentan con un alto grado de validez y confiabilidad. Cabe agregar que el AVACO-EVADIE es el único que sostiene evidencia de un estudio factorial. No obstante, no responden a algunos aspectos que son específicos del ámbito de la danza en el contexto universitario, ya que en este campo resulta difícil determinar hasta qué punto los procesos formativos concilian con la integridad física de las personas y aprueban la inclusión a la diversidad.

De este modo, con el propósito de realizar una evaluación específica sobre atención a la diversidad en estudiantes de danza se elaboró el instrumento CEADED (Cuestionario para Evaluar la Atención a la Diversidad Estudiantil en Danza), y se validó mediante un proceso de juicio de expertos y una prueba piloto. Se requiere determinar la validez de constructo y valorar su confiabilidad a través de la aplicación a una muestra mayor con la finalidad de identificar su estructura interna de manera que constituya un instrumento válido y fiable que permita evaluar la atención a la diversidad de estudiantes de danza desde un enfoque socioformativo, así como identificar con claridad qué elementos pueden mejorar para actuar en busca de soluciones y 
cambios progresivos que ayuden a modificar la cultura de los centros educativos hacia un desarrollo de las prácticas inclusivas.

Cabe precisar que aunque el tema de la inclusión y la atención a la diversidad abarca condiciones muy variadas de la humanidad (aspectos culturales, de origen, de sexo, de género, condiciones económicas, etc.), el presente estudio se enfoca particularmente en la diversidad de habilidades corporales y de condiciones físicas que pueden tener los estudiantes de danza, aspectos como, la flexibilidad, la fuerza, la coordinación y el ritmo, entre otras cualidades que atañen a la formación dancística tradicional.

Así, el presente estudio se orientó en las siguientes metas: 1) determinar la validez de constructo mediante la aplicación a un grupo de estudiantes de danza y el empleo del análisis factorial; y 2) analizar la confiabilidad y consistencia interna del instrumento.

\section{Metodología}

\section{Tipo de estudio}

Se realizó un estudio de tipo instrumental orientado a evaluar la validez de constructo y confiabilidad del cuestionario CEADED que fue construido para evaluar la atención a la diversidad en danza. Desde la perspectiva de Pacheco (2015), es importante reportar la validez y confiabilidad de un instrumento de medición para que la información recabada tenga coherencia y precisión, es decir, que el instrumento posea las condiciones y características necesarias para mostrar medidas fieles a la realidad, pues la validez de constructo valida la teoría subyancente a la medición y la confiabilidad evalúa la correlación que existe entre cada cuestión del cuestionario dentro del mismo (Dini et al., 2014).

\section{Instrumentos}

Para el estudio se emplearon dos instrumentos:

a) CEADED: Se trata de una escala tipo Likert que consta de 43 indicadores repartidos en tres dimensiones y ocho subdimensiones para ser respondida por estudiantes y en la que pueden expresar criterios personales, criterios institucionales y prácticas concretas de la comunidad estudiantil y docente.

b) Cuestionario de Factores Sociodemográficos: diseñado por el Centro Universitario CIFE (2018) para recabar datos de los participantes respecto a la edad, sexo y nivel educativo, y 
que además incluye algunos datos específicos relacionados con la trayectoria dancística y experiencia laboral.

\section{Participantes}

La muestra fue conformada de manera intencional por conveniencia por estudiantes de licenciatura en danza con edades comprendidas entre 17 y 26 años que cursaban entre el segundo y décimo semestre de la carrera en diversas universidades de la República Mexicana, restringido a las áreas de danza contemporánea, ballet clásico y danza folklórica (Tabla 1). Una parte de los participantes fueron invitados a través de una convocatoria directa a través de correo electrónico y redes sociales, y la otra de manera personal con el apoyo de colegas docentes. Los datos se tomaron durante el curso enero-julio 2019.

Tabla 1. Datos sociodemográficos de los participantes

\begin{tabular}{ll}
\hline Características & Datos \\
\hline $\mathrm{N}$ & 453 \\
a) Sexo & Mujeres $79 \%$ \\
& Hombres $21 \%$ \\
b) Edad & Media 20.64, d. e. \pm 2.7611 \\
c) Roles & Estudiantes de danza \\
d) Estado de la República & Chihuahua 41\% \\
& Nuevo León 31\% \\
& Michoacán 18\% \\
e) Área de estudios & Querétaro 10\% \\
& Danza Contemporánea 47\% \\
& Danza Clásica 30\% \\
f) Contexto & Danza Folklórica 23\% \\
\hline Elaboración propia & Universidades Públicas de México \\
\hline
\end{tabular}

\section{Fases del proceso}

a) Aplicación del instrumento a una muestra de 453 estudiantes de danza, a través de la colaboración de docentes y directamente utilizando la modalidad de formulario electrónico y cuestionario impreso.

b) Realización del análisis factorial exploratorio con el objetivo de analizar la estructura subyacente de las variables para simplificar la información que proporciona la matriz de correlaciones y facilitar su interpretación (Morales, 2013). Previamente se comprobó el 
grado de adecuación de la muestra a través del cálculo de la medida de KMO de Kaiser (1970), que como señalan Lloret-Segura, et al. (2014), es una de las formas más comunes para indicar qué tan grande es la correlación entre las variables medidas; si es lo suficientemente grande para su factorización ofrecerá resultados estables independientemente del tamaño de la muestra y el número de factores e ítems. Se suele establecer que el rango de valores del KMO es de 0 a 1 , indicando que mientras más cerca esté el valor a 1 las variables están más relacionadas entre ellas, aunque también es importante contrastar el resultado con el test de Bartlett para poner a prueba la hipótesis nula de que las variables no están correlacionadas en la población (Ferrando y AnguianoCarrasco, 2010). El siguiente paso fue verificar los valores de las comunalidades para determinar la proporción de varianza en común con el resto de las variables, considerando valores superiores a 0.40 como adecuados (Romero y Martínez, 2017). Se eligió el método de extracción de factorización de ejes principales iterado con rotación Oblimin Directo para conseguir una estructura más coherente al insertar en la matriz de correlaciones la mejor estimación posible de las comunalidades de las variables a partir de los factores retenidos (Lloret-Segura et al., 2014).

c) Evaluación de la confiabilidad y consistencia interna mediante el coeficiente de Alfa de Cronbach, que según autores como González y Pazmiño (2015) y Lao y Takakuwa (2017) es una forma sencilla para validar la correlación que existe entre los indicadores que componen la escala; señalan que los valores oscilan entre 0 y 1 , siendo los valores más cercanos a uno los que indican una mayor consistencia interna.

\section{Resultados}

\section{Validez de constructo}

Primero, se verificó que los datos obtenidos en los instrumentos cumplían con los requisitos para el análisis factorial. El índice de KMO mostró niveles adecuados de 0.913 y la prueba de Bartlett fue de $\mathrm{Xi}^{2}: 16520.819$ ( $\left.\mathrm{p}<0.001\right)$. En la Tabla 2 se indica que los valores de las comunalidades son adecuados, superiores a 0.50 en su mayoría y se representan en el modelo factorial. 
Tabla 2. Comunalidades

\begin{tabular}{|c|c|c|}
\hline & Inicial & Extracción \\
\hline VAR00001 & .671 & .635 \\
\hline VAR00002 & .733 & .677 \\
\hline VAR00003 & .692 & .712 \\
\hline VAR00004 & .780 & .804 \\
\hline VAR00005 & .769 & .723 \\
\hline VAR00006 & .771 & .733 \\
\hline VAR00007 & .707 & .683 \\
\hline VAR00008 & .690 & .539 \\
\hline VAR00009 & .764 & .765 \\
\hline VAR00010 & .767 & .709 \\
\hline VAR00011 & .750 & .669 \\
\hline VAR00012 & 639 & .607 \\
\hline VAR00013 & .663 & .499 \\
\hline VAR00014 & .724 & .733 \\
\hline VAR00015 & .698 & .607 \\
\hline VAR00016 & .738 & .625 \\
\hline VAR00017 & .752 & .647 \\
\hline VAR00018 & .673 & .541 \\
\hline VAR00019 & .781 & .613 \\
\hline VAR00020 & .777 & .639 \\
\hline VAR00021 & .726 & .670 \\
\hline VAR00022 & .800 & .767 \\
\hline VAR00023 & .775 & .655 \\
\hline VAR00024 & .756 & .694 \\
\hline VAR00025 & .706 & .582 \\
\hline VAR00026 & .627 & .473 \\
\hline VAR00027 & .668 & .573 \\
\hline VAR00028 & .800 & .664 \\
\hline VAR00029 & .666 & .509 \\
\hline VAR00030 & .725 & .617 \\
\hline VAR00031 & .735 & .634 \\
\hline VAR00032 & .745 & .654 \\
\hline VAR00033 & .679 & .613 \\
\hline VAR00034 & .786 & .651 \\
\hline VAR00035 & .794 & .690 \\
\hline VAR00036 & .689 & .610 \\
\hline VAR00037 & .762 & .824 \\
\hline VAR00038 & .689 & .605 \\
\hline VAR00039 & .787 & .784 \\
\hline VAR00040 & .674 & .710 \\
\hline VAR00041 & .653 & .565 \\
\hline VAR00042 & .702 & .696 \\
\hline VAR00043 & .693 & .663 \\
\hline
\end{tabular}

Elaboración propia 
Se extrajeron ocho factores que explican el $65.260 \%$ de la varianza total y presentan autovalores iniciales de entre 15.747 y 1.092 (Tabla 3). La nueva agrupación de los ítems resultó más acorde y simplificada.

Tabla 3. Varianza total explicada.

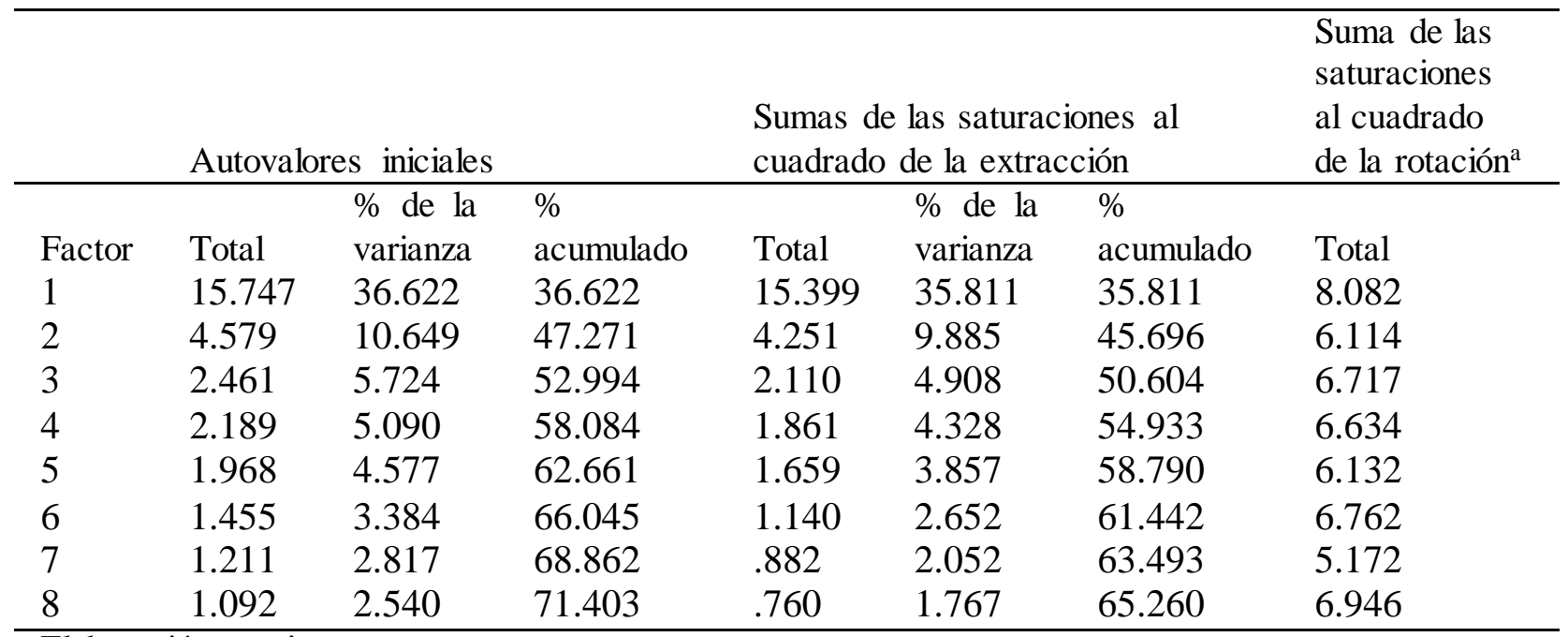

Elaboración propia

El número de factores resultantes del análisis se aproximó al número de dimensiones/subdimensiones planteadas originalmente. Los factores 1, 4, 6 y 8 se identificaron con los 24 ítems diseñados originalmente para la dimensión competencias docentes, aunque localizados en las diferentes subdimensiones que la conforman: competencias académicas, didácticas, comunicativas e integradoras y evaluadoras. El factor 2, coincidió con los 6 ítems propuestos para la subdimensión concepto; el factor 3 se identificó con los 4 ítems señalados en la dimensión concepción propia; el factor 5 se ajustó a los 5 ítems formulados en la subdimensión centro/institución; y por último el factor 7 correspondió a los 4 ítems construidos para la subdimensión currículo.

Se consideró la rotación de los factores originales para conseguir una nueva matriz factorial que funcionara mejor y facilitara la interpretación. A partir de la matriz de factores rotados, se pudo observar una clara agrupación de variables asignadas en cada factor. Con base en estos resultados y tomando en cuenta las definiciones teóricas de cada reactivo, se ajustó el nombre de cada factor para tener una idea más clara del significado y el tema que aborda cada uno (Tabla 4). 
Tabla 4. Factores $e$ items resultantes

\section{FACTOR 1. Competencias docentes}

1. Los docentes desarrollan sus clases de acuerdo a los diferentes ritmos de aprendizaje, capacidades físicas e intereses de cada estudiante.

2. Los docentes refuerzan la atención educativa a aquellos estudiantes que presentan alguna situación específica.

3. Los docentes permiten que todos los estudiantes expresen su opinión, ideas y emociones.

4. Los docentes realizan una evaluación diagnóstica previa a la clase para conocer el nivel de conocimientos de todos los estudiantes.

5. Ante estudiantes con escasas cualidades físicas para la danza, los docentes muestran incomodidad.

6. Para el desarrollo de proyectos, los docentes toman en cuenta a todos los estudiantes por igual.

7. Los docentes priorizan que se cumplan al máximo los aprendizajes prácticos y funcionales.

8. Los docentes respetan el ritmo de aprendizaje cuando un estudiante no logra ejecutar algún movimiento a la perfección.

9. Los docentes crean un clima de respeto y valoración de la diversidad entre los estudiantes.

10. Los docentes realizan prácticas fuera del aula porque consideran que son un buen complemento para el desarrollo de los estudiantes y favorece los diferentes estilos de aprendizaje.

\section{FACTOR 2. Concepción sobre diversidad.}

11. La diversidad constituye un aspecto positivo y enriquecedor para la danza.

12. La diversidad del alumnado de danza es común en nuestra institución.

13. La atención a la diversidad ocupa un papel importante en la práctica docente.

14. La diversidad nos remite al hecho de que el estudiantado tiene necesidades educativas diferentes, propias y específicas para poder acceder al aprendizaje. 
15. La diversidad se constituye por las diferencias físicas, de humor, edad, sexo, personalidad, creencias u otras que existen entre las personas.

16. Las diferentes cualidades físicas y habilidades que presenta el estudiantado puede ser un factor que retrasa el proceso de enseñanza-aprendizaje homogéneo.

\section{FACTOR 3. Interés hacia la inclusión.}

17. Estoy a favor de la inclusión en danza. Que se acepten personas con diversidad anatómica, fisiológica e intelectual.

18. La carrera en danza debe adaptarse a las nuevas generaciones.

19. El examen de admisión no debe permitir la entrada a todos los interesados, sino solo a aquellos con las cualidades físicas aptas para la danza.

20. No sugeriría la inclusión en danza porque considero que un bailarín debe tener una técnica corporal impecable además de una figura esbelta y delgada.

\section{FACTOR 4. Estrategias didácticas.}

21. Los docentes utilizan diferentes instrumentos de evaluación para valorar el progreso de cada estudiante.

22. Los docentes comprenden cuando un estudiante debe tomar un descanso, detenerse o retirarse de la clase por lesión, enfermedad o cansancio.

23. Los docentes evitan mensajes descalificadores o que impliquen situaciones comparativas entre los estudiantes.

24. Los docentes invitan a otros docentes, colegas o profesionales externos para que observen la clase y den sus comentarios a los estudiantes.

\section{FACTOR 5. La institución frente a la diversidad.}

25. La institución ofrece: cursos, talleres, conferencias u otros apoyos profesionales para reforzar la práctica encaminada a la atención a la diversidad estudiantil.

26. Las aulas de la institución están distribuidas según las necesidades concretas de cada grupo.

27. La institución promueve una organización inclusiva, mediante proyectos donde estudiantes, docentes y directivos toman decisiones en común en beneficio de la carrera. 28. La institución promueve una cultura de atención y respeto a la diversidad. 
29. Las condiciones arquitectónicas de la institución favorecen el acceso y desplazamie nto del estudiantado a todas las áreas y servicios.

\section{FACTOR 6. Competencias integradoras.}

30. Los docentes utilizan la autoevaluación como estrategia para valorar la opinión de cada estudiante sobre su propio aprendizaje.

31. Los docentes integran equipos de trabajo con estudiantes de diferentes niveles y capacidades.

32. Los docentes promueven la evaluación de resolución de problemas a partir del anális is, la reflexión, comunicación y argumentación de problemas reales.

33. Los docentes utilizan un vocabulario adecuado para dar las instrucciones y correcciones.

\section{FACTOR 7. Currículo.}

34. El currículo es flexible, permite la adaptación necesaria para hacer ajustes razonables acordes a la diversidad del alumnado.

35. Los criterios de selección para entrar a la licenciatura en danza están regidos por diversos criterios como las condiciones físicas, la creatividad y expresividad.

36. La licenciatura en danza permite el acceso a todos los interesados en el área.

37. Los estudiantes con necesidades específicas reciben la misma clase que el resto de sus compañeros.

\section{FACTOR 8. Actitud docente.}

38. Los docentes motivan a los estudiantes reforzando sus logros para que consigan superar los obstáculos, independientemente de su desempeño.

39. Los docentes facilitan la solución de conflictos mediante el diálogo.

40. Los docentes priorizan la evaluación de los logros prácticos corporales a través de exámenes bien estructurados.

41. Los docentes muestran un comportamiento excluyente con algunos estudiantes.

42. Los docentes trabajan en colaboración con otros docentes para llevar a cabo actividades académicas, investigativas, artísticas u otras. 
43. Los docentes promueven la coevaluación como estrategia para sensibilizar a los estudiantes sobre los diferentes ritmos y estilos de aprendizaje de sus compañeros.

Elaboración propia

\section{Análisis de consistencia interna}

Se presenta la confiabilidad obtenida por el coeficiente de Alfa de Cronbach, la cual reveló un valor total de 0.957 . Como puede observarse en la tabla 5, todos los factores muestran valores de alfa mayores a 0.80 , siendo el factor 5 el más elevado y el 7 el menor. En conjunto, tanto la fiabilidad total como por factores revelan una fuerte correlación entre los ítems.

Tabla 5. Resultados estadísticos de fiabilidad

\begin{tabular}{lll}
\hline & Alfa de Cronbach & N de elementos \\
\hline Factor 1. Competencias docentes. & .926 & 10 \\
Factor 2. Concepción sobre diversidad. & .899 & 6 \\
Factor 3. Interés hacia la inclusión. & .860 & 4 \\
Factor 4. Estrategias didácticas. & .858 & 4 \\
Factor 5. La institución frente a la diversidad. & .979 & 5 \\
Factor 6. Competencias integradoras. & .839 & 4 \\
Factor 7. Currículo. & .828 & 4 \\
Factor 8. Actitud docente. & .882 & 6 \\
Cuestionario completo & .957 & 43 \\
\hline
\end{tabular}

Elaboración propia

\section{Discusión}

El análisis factorial exploratorio practicado sobre el instrumento CEADED evidencia la coincidencia de la estructura factorial de ocho factores con las dimensiones consideradas en la construcción del instrumento: competencias docentes, concepción sobre diversidad, interés hacia la inclusión, estrategias didácticas, la institución frente a la diversidad, competencias integradoras, currículo y actitud docente. Las cargas factoriales corresponden en un $63 \%$ con los ítems diseñados para medir cada factor, el otro $37 \%$ de los ítems a pesar de estar localizados dentro de la dimensión 2 correspondiente al instrumento original, no tenían el mismo orden de colocación dentro de cada 
subdimensión, de modo que el análisis los repartió de diferente manera, logrando una estructura más adecuada para una mejor comprensión.

La confiabilidad del instrumento reporta un valor de Alfa de Cronbach de 0.957. Es altamente confiable para evaluar la atención a la diversidad entre estudiantes en el campo de la formación dancística profesional, brinda la posibilidad de identificar los aspectos que promueven o dificultan la atención a la diversidad del alumnado para fomentar la construcción de estrategias que impulsen una cultura de inclusión en el campo del arte danzario. Incluso, es probable que pueda adaptarse a futuro para ser aplicado a otros niveles de estudios o academias de danza.

En este sentido, el CEADED representa una propuesta innovadora para enfrentar los retos de la sociedad del conocimiento, que en concordancia con Carrillo et al. (2018), para lograr un ambiente educativo de convivencia sana y respeto a la diversidad se necesitan continuos esfuerzos de innovación pedagógica y disposición para materializar los propósitos. Por lo tanto, significa una invitación al cambio en el marco de la sociedad del conocimiento con base en los principios de la socioformación y el desarrollo sostenible.

El CEADED muestra similitud con los instrumentos ACADI (Arnaiz y Guirao, 2015), AVACO-EVADIE (Biencinto-López et al., 2009) y CEEIU (De la Herrán et al., 2017) en cuanto que aporta un sistema de indicadores útiles y confiables para evaluar tanto la atención a la diversidad dentro del marco de la inclusión educativa, como identificar aquellos factores que limitan la atención a la diversidad y señalar situaciones que sean susceptibles de mejora.

Por lo tanto, tomando como referencia que en el campo de la danza existe un gran vacío de instrumentos para el estudio de la diversidad e inclusión, haber realizado el análisis factorial del CEADED es uno de los mayores logros, pues a pesar de ser pocos los instrumentos encontrados para evaluar el constructo "atención a la diversidad", de manera general en la educación son demasiado pocos los que sustentan una validez de constructo tras haber sido sometidos a anális is factorial. El CEADED además de resaltar que por primera vez se estudia el tema con base en los principios de la socioformación, obtuvo valores significantes en el estudio factorial.

Algo que llama la atención, es que la mayoría de estos instrumentos buscan conocer la opinión del personal docente, administrativo y de apoyo, mas no del alumnado, a excepción del CEEIU que evalúa el constructo "educación inclusiva universitaria" y busca conocer además de la opinión del equipo docente y directivo, la del estudiantado. Es importante exaltar entonces, el fomento de la equidad e igualdad como parte fundamental para favorecer la construcción de una sociedad más 
justa y sensible con tendencia a incorporar nuevas formas de enfrentar la educación actual (Domínguez, et al., 2015).

Finalmente, de acuerdo con Carvajal, et al. (2011), el proceso de validación de un instrume nto es continuo, de manera que mientras más propiedades psicométricas se hayan medido en distintos contextos y sujetos, mayor consistencia va adquiriendo y permite construir nuevas predicciones. Por ello, se sugiere su aplicación en otras muestras para ratificar su consistencia interna y contribuir a entender de mejor forma el constructo del instrumento. Además, será interesante realizar un estudio correlacional entre poblaciones nacionales y extranjeras.

\section{Referencias}

Agreda, M., Hinojos, M. A., y Sola, J. M. (2016). Diseño y validación de un instrumento para evaluar la competencia digital de los docentes en la educación superior española. Revista de Medios y Educación, 49, 39-56. DOI: http://dx.doi.org/10.12795/pixelbit.2016.i49.03

Arnaiz, P. y Guirao, J. M. (2015). La autoevaluación de centros en España para la atención a la diversidad desde una perspectiva inclusiva. Revista Electrónica Interuniversitaria de Formación del Profesorado, 18(1), 45-101. DOI: https://doi.org/10.6018/reifop.18.1.214341

Biencinto-López, Ch., González-Barbera, C., García-García, M., Sánchez-Delgado, P., y MadridVivar, D. (2009). Diseño y propiedades psicométricas del AVACO-EVADIE. Cuestionario para la evaluación de la atención a la diversidad como dimensión educativa en las instituciones escolares. Relieve, 15(1), 1-36. Recuperado de: http://www.uv.es/RELIEVE/v15n1/RELIEVEv15n1_4.htm.

Brozas-Polo, M. y Vicente-Pedraz, M. (2017). La diversidad corporal en la danza contemporánea: una mirada retrospectiva al siglo XX. Arte, Individuo y Sociedad, 29(1), 71-87. Recuperado de: https://dialnet.unirioja.es/servlet/articulo?codigo=5894795

Carvajal A., Centeno C., Watson R., Martínez M., y Sanz A. (2011). ¿Cómo validar un instrumento de medida de la salud? Anales del sistema Sanitario de Navarro, 34(1), 63-72. Recuperado de: http://scielo.isciii.es/scielo.php?script=sci_arttextypid=S1137-66272011000100007

Carrillo, S. M., Forgiony, J. O., Rivera, D. A., Bonilla, N. J., Montanchez, M. L., y Alarcón, M. F. (2018). Prácticas pedagógicas frente a la educación inclusiva desde la perspectiva del 
docente. Revista Espacio, 39(17), 15-32. Recuperado de: http://www.revistaespacios.com/a18v39n17/18391715.html

CIFE. (2018). Instrumento "Factores Sociodemográficos". México. Centro Universitario CIFE. Recuperado de: https://cife.edu.mx/recursos/

De la Herrán G., Paredes, J., y Monsalve, D. V. (2017). Cuestionario para la evaluación de la educación inclusiva universitaria (CEEIU). Revista Complutense de Educación, 28(3), 913928. Recuperado de: http:/hdl.handle.net/10486/680796

Dini, A. P., Alves, D. F., Oliveira, H. C., y Guirardello, E. B. (2014). Validez y confiabilidad de un instrumento de clasificación de pacientes pediátricos. Revista Latino-Americana de Enfermagem, 22(4), 598-603. DOI: https://dx.doi.org/10.1590/0104-1169.3575.2457

Domínguez, J., López, A., Pino, M., y Vázquez, E. (2015). Integración o inclusión: El dilema educativo en la atención a la diversidad. Revista Portuguesa de Educación, 28(2), 3150. Recuperado de: http://www.redalyc.org/pdf/374/37443385003.pdf

Ferrando, P. J. y Anguiano-Carrasco (2010). El análisis factorial como técnica de investigación en Psicología. Papeles del Psicólogo, 31(1), 18-33. Recuperado de: http://www.papelesdelpsicologo.es/pdf/1793.pdf

González, A. J. y Pazmiño, S. M. (2015). Cálculo e interpretación del Alfa de Cronbach para el caso de validación de la consistencia interna de un cuestionario, con dos posibles escalas tipo Likert. Revista Publicando, 2(1), 62-67. Recuperado de: https://nbnresolving.org/urn:nbn:de:0168-ssoar-42382

Kaiser, H. F. (1970). A second-generation little jiffy. Psychometrika, 35(4), 401-415. Recuperado de: https:/link.springer.com/article/10.1007\%2FBF02291817?LI=true

Lao, T. y Takakuwa, R. (2017). Análisis de confiabilidad y validez de un instrumento de medición de la sociedad del conocimiento y su dependencia en las tecnologías de la información y comunicación. Revista de Iniciación Científica, 2(2), 64-75. Recuperado de: https://n9.cl/YPZ6

Lloret-Segura, S., Ferreres-Traver, A., Hernández-Baeza, A., y Tomás-Marco, I. (2014). El anális is factorial exploratorio de los ítems: una guía práctica, revisada y actualizada. Anales de Psicología, 30(3), 1151-1169. DOI: http://dx.doi.org/10.6018/analesps.30.3.199361

Montes, V. M. (2017). La danza contemporánea como estrategia comunicacional para la inclusión social de personas con discapacidad física: el caso de Kinesfera Danza (Tesis de 
licenciatura). Pontifica Universidad Católica del Perú, Lima, Perú. Recuperado de: https://core.ac.uk/download/pdf/96366256.pdf

Morales, P. (2013). El análisis factorial en la construcción e interpretación de test, escalas y cuestionarios. Universidad Pontificia Comillas, Madrid. Recuperado de: https://web.upcomillas.es/personal/peter/investigacion/AnalisisFactorial.pdf

Niño, N., y Tobón, S. (2017). El plan de mejora de la unidad de aprendizaje patrimonio natural y turismo desde la socioformación. Universidad y Ciencia, 6, 98-114. Recuperado de: http://revistas. unica.cu/index.php/uciencia/article/view/704/0

Pacheco, P. R. (2015). Construcción y validación de los instrumentos para la medición de la influencia de los campos emocionales en los aprendizajes significativos. Revista Internacional de Educación y Aprendizaje, 3(1), 1-24. Recuperado de: https://journals.epistemopolis.org/index.php/educacion/issue/download/102/258

Ramos-Carranza, I., Medina-Rodríguez, R., Morales-Sánchez, V., Morquecho, R., y Ceballos, O. (2015). La gestión efectiva de instalaciones deportivas públicas: diseño de un instrumento. Revista Iberoamericana de Psicología del Ejercicio y el Deporte, 10(2), 285-291. Recuperado de: https://sudocument.ulpgc.es/bitstream/10553/13606/1/0537108_20152_0014.pdf

Romero, T., y Martínez, A. (2017). Construcción y validación de un cuestionario de evaluación al desempeño docente mediante Análisis Factorial Exploratorio. Revista Científica De FAREM-Estelí, (22), 18-30. DOI: https://doi.org/10.5377/farem.v0i22.4514

Severiche-Sierra, C., Gómez-Bustamante, E., y Jaimes-Morales, J. (2016): La educación ambiental como base cultural y estrategia para el desarrollo sostenible. Telos, 18(2): 266-281. https://www.redalyc.org/pdf/993/99345727007.pdf

Tobón, S. (2017). Conceptual analysis of the socioformation according to the knowledge society. Knowledge Society and Quality of Life (KSQL), 1(1), 9-35. https://goo.gl/aJeSvw 